\title{
Nosocomial Respiratory Infections in a Rural Zambian Hospital
}

\author{
Gideon Loevinsohn, ${ }^{1,2}$ Justin Hardick, ${ }^{3}$ Thomas Mehoke, ${ }^{4}$ Pamela Sinywimaanzi, ${ }^{5}$ Mutinta Hamahuwa, ${ }^{5}$ \\ Katherine Z. J. Fenstermacher, ${ }^{6}$ Kathryn Shaw-Saliba, ${ }^{6}$ Peter Thielen, ${ }^{4}$ Jared Evans, ${ }^{4}$ Kenneth Bowden, ${ }^{4}$ Kristina Zudock, ${ }^{4}$ \\ Lauren M. Sauer, ${ }^{6}$ Mwaka Monze, ${ }^{7}$ Charlotte A. Gaydos, ${ }^{3}$ Richard E. Rothman, ${ }^{6}$ Andrew Pekosz, ${ }^{8}$ Philip E. Thuma, ${ }^{5,8}$ and \\ Catherine G. Sutcliffe 1* $^{*}$ \\ ${ }^{1}$ Department of Epidemiology, Johns Hopkins University Bloomberg School of Public Health, Baltimore, Maryland; ${ }^{2}$ Johns Hopkins School of \\ Medicine, Baltimore, Maryland; ${ }^{3}$ Division of Infectious Diseases, Johns Hopkins University School of Medicine, Baltimore, Maryland; ${ }^{4}$ Applied \\ Physics Laboratory, Johns Hopkins University, Laurel, Maryland; ${ }^{5}$ Macha Research Trust, Choma, Zambia; ${ }^{6}$ Department of Emergency Medicine, \\ Johns Hopkins University School of Medicine, Baltimore, Maryland; ${ }^{7}$ Virology Laboratory, University Teaching Hospital, Lusaka, Zambia; \\ ${ }^{8}$ Department of Molecular Microbiology and Immunology, Johns Hopkins University Bloomberg School of Public Health, Baltimore, Maryland
}

\begin{abstract}
The burden of nosocomial respiratory infections in rural southern Africa is poorly understood. We established a surveillance program at a rural Zambian hospital to detect influenza-like illness (ILI) and respiratory infections among hospitalized patients and a cohort of healthcare workers (HCWs). Nasopharyngeal specimens from symptomatic patients and HCWs underwent broadly multiplexed molecular testing to detect viruses and atypical bacteria. During 1 year of surveillance, 15 patients (1.7\% of admissions) developed ILI more than 48 hours after admission. Among $44 \mathrm{HCWs,} 19$ (43\%) experienced at least one ILI episode, with a total of $31 \mathrm{ILI}$ episodes detected. Respiratory viruses were detected in $45 \%$ of patient and $55 \%$ of HCW specimens. The cumulative incidence of influenza infection among HCWs over 1 year was $9 \%$. Overall, respiratory viruses were commonly found among patients and $\mathrm{HCWs}$ in a rural Zambian hospital with limited infection control infrastructure.
\end{abstract}

Respiratory viruses are efficiently transmitted within inpatient populations, and nosocomial respiratory infections are associated with higher costs and case-fatality rates than community-acquired disease. ${ }^{1,2}$ Within hospitals, healthcare workers (HCWs) often contribute to viral transmission. Although influenza vaccination of HCWs has reduced nosocomial viral transmission in some high-income settings, influenza vaccines are not widely available in many low- and middleincome countries, including most countries in southern Africa. $^{3}$

The burden of nosocomial respiratory infections in southern Africa is poorly understood with few established surveillance systems. This is concerning because facilities are frequently overcrowded, with open wards, scarce personal protective equipment, and limited infection control infrastructure. Rural facilities, which serve the majority of the region's population, ${ }^{4}$ have been particularly understudied. These institutions have fewer resources than urban hospitals and may serve as hubs for the spread of high-risk pathogens. ${ }^{5}$ These concerns are especially relevant given the ongoing spread of severe acute respiratory syndrome coronavirus 2 (SARS-CoV-2), which is currently causing significant morbidity, mortality, and resource shortages in the region and within Zambia. ${ }^{6}$

We implemented surveillance for nosocomial respiratory infections at a hospital in rural Zambia and a prospective cohort study of HCWs to characterize the burden of respiratory infections within the hospital setting.

Macha Hospital is a 208-bed, district-level facility located in a rural region of Southern Province and serves as a referral hospital for smaller hospitals and rural health centers in its catchment area. Acute respiratory infections are the leading cause of hospital presentation, with respiratory viruses detected in the majority of patients with respiratory symptoms. $^{7}$ Macha hospital has limited infection-control

\footnotetext{
* Address correspondence to Catherine Sutcliffe, Johns Hopkins Bloomberg School of Public Health, 615 N. Wolfe Street, Room E6535, Baltimore, MD 21205. E-mail: csutcli1@jhu.edu
}

infrastructure, and inpatient care is provided in seven open wards. Inpatients are typically accompanied by family members, who provide care during the day and stay overnight in crowded encampments nearby.

Patients on the male, female, and pediatric wards were monitored twice daily through medical record review and patient self-report. Eligible patients included those admitted for nonrespiratory complaints who developed influenza-like illness (ILI) $>48$ hours after admission. Nosocomial ILI was defined as documented $\left(\geq 38^{\circ} \mathrm{C}\right.$ ) or reported fever with cough or sore throat with onset or clinical worsening $>48$ hours postadmission. After obtaining written informed consent, study staff ascertained clinical symptoms, hospital-based exposures, and medical history through medical record review and patient interviews and measured peripheral capillary oxygen saturation $\left(\% \mathrm{SpO}_{2}\right)$ using a handheld pulse oximeter. $\mathrm{A}$ nasopharyngeal specimen was collected with a flocked swab, placed in universal transport media, and transported to the Macha Research Trust Clinical Research Laboratory in Macha.

A cohort of hospital employees $\geq 18$ years of age with direct patient contact was established. At enrollment, consenting HCWs completed a questionnaire detailing demographic information, medical history, and occupational exposures. Participants were followed weekly to ascertain the development of ILI. If ILI was reported, an in-person visit was conducted to assess symptoms and exposures, measure temperature and oxygen saturation, and collect a nasopharyngeal swab. At one visit, HCWs were asked if they would be willing to receive the seasonal influenza vaccine if it were made available at no charge.

Nasopharyngeal samples from both study populations were tested using the Xpert Xpress Flu/RSV assay (Cepheid Inc., Sunnyvale, CA) at the Clinical Research Laboratory and the FilmArray Respiratory Panel EZ (BioFire Diagnostics, Salt Lake City, UT) at Johns Hopkins University in Baltimore, MD, to detect common respiratory viruses and atypical respiratory bacteria as previously described. ${ }^{7}$ Influenza virus-positive specimens were genetically sequenced at the Johns Hopkins 
Applied Physics Laboratory to establish subtype based on the hemagglutinin sequences. ${ }^{8}$

The cumulative incidence and incidence rate of nosocomial infections were determined among eligible inpatients. Poisson regression was used to compare incidence rates by group. After exploratory data analysis, admission diagnosis was dichotomized as orthopedic (includes fractures, joint dislocations, and amputations) versus nonorthopedic.

Tachypnea was defined based on age-specific values. ${ }^{9}$ Hypoxemia was defined as an $\mathrm{SpO}_{2} \leq 92 \%$. Ward occupancy was defined as the proportion of beds within a ward currently occupied. For influenza and respiratory syncytial virus, participants testing positive on either test were defined as infected. Clusters of infections were defined as multiple patients or HCWs with epidemiological links who developed ILI within a 7-day window and tested positive for the same pathogen.

From December 10, 2018 to December 9, 2019, 1,365 patients were admitted for nonrespiratory complaints (76\% of all admissions). The mean duration of admission was 4 days, and 871 patients (64\%) were admitted for $>48$ hours. Patients admitted for $>48$ hours were monitored over 4,706 inpatient-days. Fifteen patients (1.7\%) developed nosocomial ILI (0.32 infections/100 inpatient-days).

The likelihood of nosocomial infection varied by ward and admission diagnosis. The incidence was higher for pediatric
(3.0\%; 0.52 infections $/ 100$ inpatient-days) than adult inpatients $(0.5 \% ; 0.09$ infections $/ 100$ inpatient-days), although this difference was not statistically significant (incidence rate ratio: 3.88; 95\% confidence interval: 0.84-17.96). The incidence was significantly higher for patients admitted for orthopedic injuries $(4.6 \%$; $0.59 / 100$ inpatient-days) than for other complaints $(0.9 \% ; 0.17 / 100$ inpatient-days; incidence rate ratio: $3.44 ; 95 \%$ confidence interval: $1.01-11.75)$.

Of the 15 patients with nosocomial ILI, 11 (73\%) were enrolled (Table 1). The median time from admission to ILI onset was 9 days (interquartile range: 3-13). Four nosocomial cases (36\%) were pediatric patients hospitalized for bone fractures and kept in traction. Two nosocomial cases died during the hospitalization (recorded causes of death were stroke and burn injury).

A pathogen was detected in five samples (45\%) from nosocomial cases (Figure 1); three patients (27\%) were infected with influenza $A$, and two patients (18\%) were infected with rhinovirus. Of the two cases who died, a pathogen (rhinovirus) was detected in one specimen (50\%).

Forty-four HCWs were enrolled and followed over the same time period as the inpatient ILI surveillance (Table 1). Only one $\mathrm{HCW}$ reported any lifetime influenza vaccination, and none reported vaccination in the prior year. Nevertheless, 37 of 43 HCWs (86\%) reported being willing to accept influenza vaccination if made available free of charge.

TABLE 1

Characteristics of inpatients and healthcare workers

\begin{tabular}{|c|c|c|}
\hline & Inpatients $(N=11)$ & Healthcare workers $(N=44)$ \\
\hline Age, years; median (IQR) & $7.0(4.0-11.0)$ & $29.0(25.0-35.5)$ \\
\hline Female, $n(\%)$ & $3(27)$ & $26(59)$ \\
\hline \multicolumn{3}{|l|}{ Inpatient ward, $n(\%)$} \\
\hline Male ward & $1(9)$ & - \\
\hline Female ward & $1(9)$ & - \\
\hline Pediatric ward & $9(82)$ & - \\
\hline \multicolumn{3}{|l|}{ Primary admission diagnosis, $n(\%)$} \\
\hline Bone fracture & $4(36)$ & - \\
\hline Asthma exacerbation & $1(9)$ & - \\
\hline Acute gastroenteritis & $1(9)$ & - \\
\hline Burns & $1(9)$ & - \\
\hline Congestive heart failure exacerbation & $1(9)$ & - \\
\hline Hepatitis & $1(9)$ & - \\
\hline Pulmonary tuberculosis & $1(9)$ & - \\
\hline Sickle cell crisis & $1(9)$ & - \\
\hline Ward occupancy \%, median (IQR) & $43(24-83)$ & - \\
\hline Use of respiratory devices, ${ }^{*} n(\%)$ & $4(36)$ & - \\
\hline Accompanied by caregiver while in hospital, $\uparrow n(\%)$ & $8(73)$ & - \\
\hline Time from admission to symptom onset, days; median (IQR) & $9(3-13)$ & - \\
\hline Duration of admission, days; median (IQR) & $12(6-22)$ & - \\
\hline Died in hospital, $n(\%)$ & $2(18)$ & - \\
\hline \multicolumn{3}{|l|}{ Occupation, $n(\%)$} \\
\hline Physician & - & $1(2)$ \\
\hline Clinical officer & - & $6(14)$ \\
\hline Nurse & - & $28(64)$ \\
\hline Midwife & - & $2(5)$ \\
\hline Other & - & $7(16)$ \\
\hline \multicolumn{3}{|l|}{ Primary location with patient contact, $n(\%)$} \\
\hline Male ward & - & $6(14)$ \\
\hline Female ward & - & $7(16)$ \\
\hline Pediatric ward & - & $8(18)$ \\
\hline Outpatient department, & - & $17(39)$ \\
\hline Otherł & - & $6(14)$ \\
\hline Ever received influenza vaccination, $n$ (\%) & - & $1(2)$ \\
\hline Received influenza vaccination in the past year, $n(\%)$ & - & $0(0)$ \\
\hline Weeks of follow up; median (IQR) & - & $46(40-50)$ \\
\hline
\end{tabular}

$\mathrm{IQR}=$ interquartile range.

* Includes nasal cannulae, respiratory masks, nebulizers, and endotracheal or nasogastric tubes.

† Refers to family member or friend who has helped to provide care while inpatient.

‡ Includes HIV testing counsellors, clinical dentists, pharmacists, medical assistants, and nurses working in the maternity ward. 


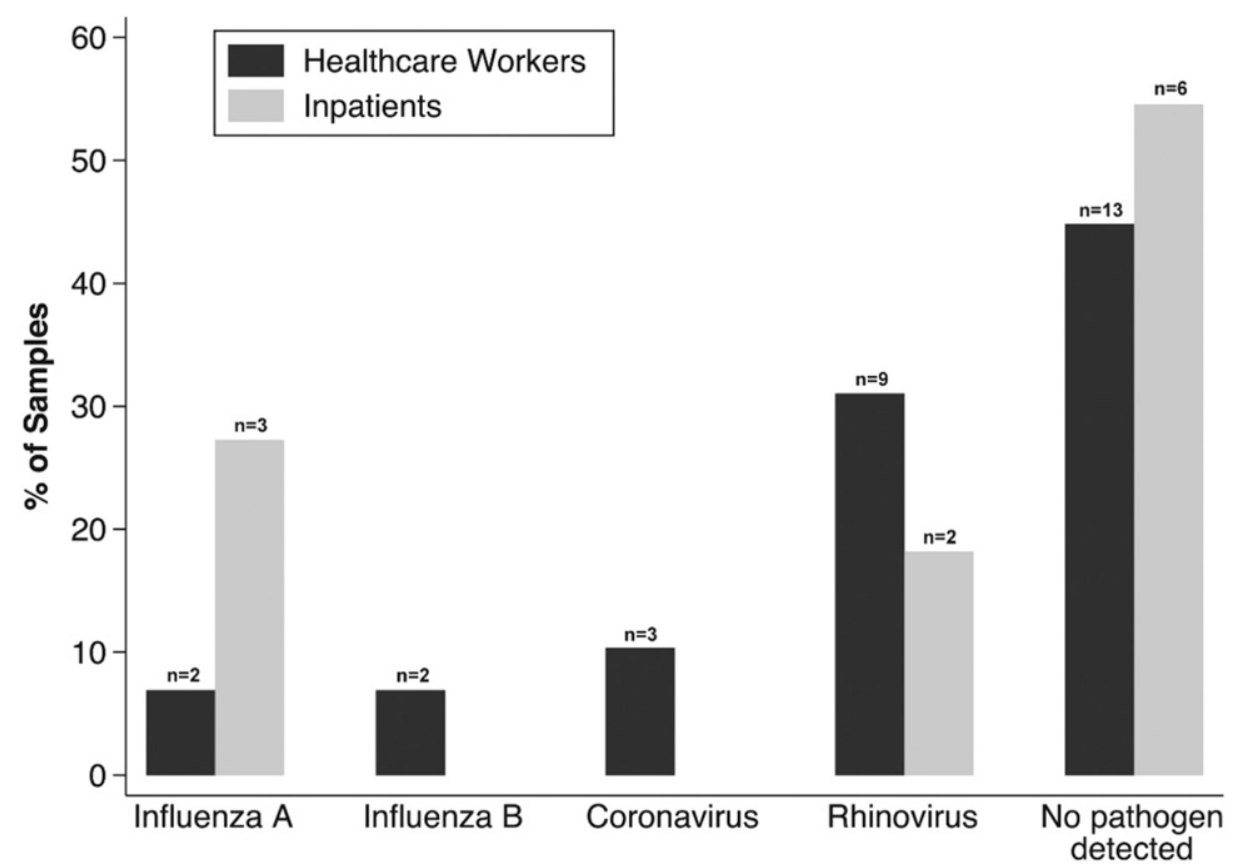

FIGURE 1. Respiratory pathogens among healthcare workers and hospitalized patients. Percentages refer to proportion of total samples within each study population. Assays included antigenic targets for the following pathogens: adenovirus, coronavirus (non-SARS-CoV-2 species: HKU1, 229E, OC43, and NL63 subtypes), human metapneumovirus, rhinovirus/enterovirus, influenza A virus, influenza B virus, parainfluenza virus, respiratory syncytial virus, Bordetella pertussis, Chlamydia pneumoniae, and Mycoplasma pneumoniae.

Over 1,818 provider-weeks of follow-up, $31 \mathrm{ILI}$ episodes were detected (1.7 per 100 provider-weeks). Nineteen HCWs (43\%) reported at least one episode of ILI (range: 1-3 episodes). Episodes of ILI were reported throughout the year, with the highest monthly incidence in June and August (Supplemental Figure 1). This corresponded to the yearly peak in adult ILI prevalence in the wider patient population (data not shown).

Only $30 \%$ (9 of 30 ) of symptomatic HCWs reported staying home from work due to their illness. Among these, the median number of workdays missed was 2 (interquartile range: 1-2).

Nasopharyngeal specimens were obtained for 29 of 31 ILI episodes among HCWs. A pathogen was detected in 16 of 29 specimens (55\%; Figure 1). No coinfections were detected. Rhinovirus was the most commonly detected pathogen (9 of 29 specimens; 31\%). Four cases of influenza (two influenza A H3N2 [7\%] and two influenza B [7\%]) and three cases of non-SARS-CoV-2 coronavirus infection (10\%) were detected. Over the 1-year study period, therefore, $9 \%$ of HCWs (4/44) experienced symptomatic influenza infections.

Based on microbiological testing results, one cluster of nosocomial influenza virus infections was detected. In August 2019, two pediatric inpatients developed ILI after admission and were found to be infected with influenza H3N2 viruses. The patients' beds were adjacent to one another in an open ward. During the period spanning the time from the admission of the first patient to the development of illness in the second patient, 11 other patients newly admitted with respiratory symptoms to the ward tested positive for influenza A H3N2. This included three patients with beds adjacent to at least one of the nosocomial cases. There were no observed clusters of infections involving HCWs and no apparent association of $\mathrm{HCW}$ infections with the observed nosocomial cluster.

Here, we report on 1 year of surveillance for healthcareassociated respiratory infections in rural Zambia. We found a cumulative annual incidence of inpatient nosocomial infections of $1.7 \%$, which is similar to that reported among studies using broad acute respiratory infection definitions in nonoutbreak, urban, low- and middle-income settings, with estimates ranging from $0.9 \%$ in Nigeria ${ }^{10}$ to $1.7 \%$ in Bangladesh. ${ }^{11}$ Patients with orthopedic injuries, and particularly those in prolonged traction, appear to be at increased risk of nosocomial infection. Immobilization, such as through traction and prolonged hospital stays, are well-established risk factors for hospital-acquired infections. ${ }^{12}$

We found a cumulative annual incidence of symptomatic influenza of $9 \%$ among HCWs. This is consistent with estimates from serologic studies among unvaccinated HCWs in Europe and North America, where incidence ranged from $1.5 \%{ }^{13}$ to $12.4 \% .{ }^{14}$ However, the observed cumulative annual incidence observed is likely an underestimate because viral testing was based on self-reported ILI symptoms that correlate poorly with serological evidence of influenza infection. ${ }^{15}$ Few HCWs reported staying home from work due to their illness, setting the stage for onward transmission both among HCWs and from HCWs to patients. Notably, most HCWs were willing to adopt seasonal influenza vaccination if made available to them. Further study is needed to understand whether vaccination of HCWs is cost effective in this setting based on local viral epidemiology, economic burden, and costs associated with distribution. ${ }^{16}$

This study is not without limitations. First, although the ILI definition used in this study is commonly used for detecting 
influenza, the requirement of fever makes it less sensitive for viral infections that may present without fever. ${ }^{17}$ Second, symptoms among inpatients and self-reporting HCWs may have been underreported, resulting in missed ILI cases. Finally, patients infected while hospitalized but manifesting symptoms after discharge were missed.

In a rural Zambian setting, there was evidence of significant in-hospital transmission of respiratory viruses. In the midst of a pandemic, such as with SARS-CoV-2, factors promoting nosocomial transmission are likely to be exacerbated and require focused surveillance efforts to inform interventions. Contextspecific infection-control approaches are needed to address these challenges.

Received November 13, 2020. Accepted for publication April 25, 2021.

Published online July 19, 2021.

Note: Supplemental figure appears at www.ajtmh.org.

Financial support: The work was supported by the National Institute of Allergy and Infectious Diseases contract HHSN272201400007C awarded to the Johns Hopkins Center of Excellence in Influenza Research and Surveillance (JHCEIRS) at the Johns Hopkins University. Any opinions, findings, conclusions, or recommendations expressed in this publication are those of the authors and do not represent the policy or position of NIAID or NIH. The funding agency had no role in the analysis of the data, writing of the manuscript, or the decision to submit the manuscript for publication.

Authors' addresses: Gideon Loevinsohn and Catherine G. Sutcliffe, Johns Hopkins University Bloomberg School of Public Health, Epidemiology, Baltimore, MD, E-mails: gloevin1@jhmi.edu and csutcli1@jhu. edu. Justin Hardick and Charlotte A. Gaydos, Johns Hopkins School of Medicine, Medicine, Infectious Diseases, Baltimore, MD, E-mails: jhardic1@jhmi.edu and cgaydos@jhmi.edu. Thomas Mehoke, Johns Hopkins, Applied Physics Laboratory, Baltimore, MD, E-mail: thomas.mehoke@jhuapl.edu. Pamela Sinywimaanzi, Macha Research Trust, Influenza Study, Choma, Zambia, E-mail: pamela. sinywimaanzi@macharesearch.org. Mutinta Hamahuwa, Macha Research Trust, Clinical Research Laboratory, Choma, Southern Province, Zambia, E-mail: mutintahamahuwa@gmail.com. Katherine Z. J. Fenstermacher, Richard E. Rothman, and Lauren M. Sauer, Johns Hopkins School of Medicine, Emergency Medicine, Baltimore, MD, E-mails: kfenste1@jhu.edu, rrothma1@jhmi.edu, and Isauer2@ jhmi.edu. Kathryn Shaw-Saliba, National Institutes of Health, National Institute of Allergy and Infectious Diseases, Bethesda, MD, E-mail: kshawsaliba@gmail.com. Peter Thielen, Jared Evans, Kenneth Bowden, and Kristina Zudock, Johns Hopkins University, Applied Physics Laboratory, Laurel, MD, E-mails: peter.thielen@jhuapl.edu, jared.evans@jhuapl.edu, kbowden2@jhmi.edu, and kzudock2@jhu. edu. Mwaka Monze, National Influenza Centre, Virology Laboratory, Lusaka, Lusaka, Zambia, E-mail: mwakamonze@hotmail.com. Andrew Pekosz, Johns Hopkins University Bloomberg School of Public Health, Molecular Microbiology and Immunology, Baltimore, MD, E-mail: apekosz1@jhu.edu. Philip E. Thuma, Macha Research Trust, Clinical Research Department, Choma, Zambia, E-mail: phil. thuma@macharesearch.org.

\section{REFERENCES}

1. Jacobs P, Lier D, Gooch K, Buesch K, Lorimer M, Mitchell I, 2013. A model of the costs of community and nosocomial pediatric respiratory syncytial virus infections in Canadian hospitals. Can J Infect Dis Med Microbiol 24: 22-26.

2. Álvarez-Lerma F, Marín-Corral J, Vilà C, Masclans JR, Loeches IM, Barbadillo S, González De Molina FJ, Rodríguez A, 2017. Characteristics of patients with hospital-acquired influenza A (H1N1)pdm09 virus admitted to the intensive care unit. $J$ Hosp Infect 95: 200-206.

3. Ortiz JR et al., 2016. A global review of national influenza immunization policies: analysis of the 2014 WHO/UNICEF Joint Reporting Form on immunization. Vaccine 34: 5400-5405.

4. World Bank, 2018. World Bank Open Data: Rural Population. Available at: https://data.worldbank.org/indicator/SP.RUR. TOTL.ZS. Accessed May 28, 2020.

5. Gandhi NR et al., 2013. Nosocomial transmission of extensively drug-resistant tuberculosis in a rural hospital in South Africa. $J$ Infect Dis 207: 9-17.

6. Mwananyanda L et al., 2021. Covid-19 deaths in Africa: prospective systematic postmortem surveillance study. BMJ 372: n334.

7. Loevinsohn $\mathrm{G}$ et al., 2020. Respiratory pathogen diversity and co-infections in rural Zambia. IJID 102: 291-298.

8. Burke DF, Smith DJ, 2014. A recommended numbering scheme for influenza A HA subtypes. PLoS One 9: e112302.

9. WHO, 2008. Integrated Management of Childhood Illness for High HIV Settings. Available at: http://whqlibdoc.who.int/ publications/2008/9789241597388_eng.pdf. Accessed April 10, 2020.

10. Daniel SO, 1977. An epidemiological study of nosocomial infections at the Lagos University Teaching Hospital. Public Health 91: 13-18.

11. Gurley ES, Zaman RU, Sultana R, Bell M, Fry AM, Srinivasan A, Rahman M, Rahman MW, Hossain MJ, Luby SP, 2010. Rates of hospital-acquired respiratory illness in Bangladeshi tertiary care hospitals: results from a low-cost pilot surveillance strategy. Clin Infect Dis 50: 1084-1090.

12. Centers for Disease Control and Prevention, 1994. Guideline for prevention of nosocomial pneumonia. Respir Care 39: 11911236.

13. Kumpulainen V, Mäkelä M, 1997. Influenza vaccination among healthy employees: a cost-benefit analysis. Scand $\mathrm{J}$ Infect Dis 29: 181-185.

14. Keitel WA, Cate TR, Couch RB, Huggins LL, Hess KR, 1997. Efficacy of repeated annual immunization with inactivated influenza virus vaccines over a five year period. Vaccine 15: 1114-1122.

15. Elder AG, O'Donnell B, McCruden EA, Symington IS, Carman WF, 1996. Incidence and recall of influenza in a cohort of Glasgow healthcare workers during the 1993-4 epidemic: results of serum testing and questionnaire. BMJ 313: 1241-1242.

16. Newall AT, Chaiyakunapruk N, Lambach P, Hutubessy RCW, 2018. WHO guide on the economic evaluation of influenza vaccination. Influenza Other Respir Viruses 12: 211-219.

17. Chughtai AA, Wang Q, Dung TC, Macintyre CR, 2017. The presence of fever in adults with influenza and other viral respiratory infections. Epidemiol Infect 145: 148-155. 